\title{
Application of Coagulation + Hydrolytic acidification+Tubular Membrane Bioreactor(MBR) System in Printing and Dyeing Wastewater Treatment
}

\author{
Huawei Sun, Yufeng Zhang (Corresponding author), Zhaohui Zhang \& Heng Zhao \\ Key Laboratory of Hollow Fiber Membrane Materials and Membrane Process \\ Tianjin Polytechnic University, Tianjin 300160, China \\ E-mail: zyf9182@tjpu.edu.cn \\ Enhua Liu \\ Motimo Group of Tianjin Polytechnic University, Tianjin 300160, China \\ E-mail: Liu-Enhua@sohu.com
}

\begin{abstract}
In the present paper, application of tubular MBR process in printing and dyeing wastewater treatment was investigated. Results showed that polyaluminium chloride(PAC) was the best coagulant; When MLSS=8g/L and HRT=8h, removal of COD and chroma was $82 \%$ and $80 \%$ in tubular MBR, respectively. Removal of organic pollutants mainly depended on biological degradation, and retention of membrane just enhanced the removal efficiency of MBR. Effects of sludge concentration on the overall attenuation of membrane flux was little, but on the initial flux was evident.
\end{abstract}

Keywords: Printing and dyeing wastewater, MBR, Tubular membrane

\section{Introduction}

The quality and quantity of printing and dyeing wastewater varied greatly with high chroma and complicated components, which was the difficulty in industrial wastewater treatment(Yang, 2002). Recently, traditional process of "hydrolytic acidification+ aerobic contact oxidation" have been extensively applied in wastewater treatment(Min, 2003, PP.16-19; Wang, 2006, PP. 54-55; Chen, 2006, PP.92-94; Zheng, 1998, PP.22-23), however, such process not only occupied large area, but also its effluents were tough to attain the treatment requirement. Therefor, in the present paper, we put forward a new process for printing and dyeing wastewater treatment, viz. Coagulation +Hydrolytic acidification+Tubular MBR System. Compared to immersed MBR applied extensively at present, pollution resistance of tubular MBR was high with big membrane flux, and drag damage of membrane module was little and easy to rinse. The phenomena that quality of effluents was attenuated due to broken wire in the immerse MBR would not appear(Malpei, 2003, PP.33-39; Gao, 2008, PP.518-520; Stephenson, 2000; Hao, 2005, PP.77-80). Studies on the application of tubular MBR system in printing and dyeing wastewater treatment were less available, therefor, tubular MBR process in printing and dyeing wastewater treatment was investigated with emphasis on the removal of pollutants in the present paper. Meanwhile, in the present process stable efficacy was obtained in wastewater treatment, and the quality of effluents completely conformed to the first grade discharge standard of textile industrial pollutants.

\section{Trial}

\subsection{Experimental water and sludge source}

Sludge used for our trial were all sampled from aeration tank mixture in Tianjin Jizhuangzi wastewater treatment factory, and original water was obtained by artificial simulation of water distribution. Original water quality was listed in detail in Table 1.

\subsection{Experimental apparatus}

Operation manner of this trial was continuous and dynamic treatment, and its water quantity was $1 \mathrm{~L} / \mathrm{h}$. Trail technological process was depicted in Figure 1. Separable MBR bioreactor was used in the experiment, and membrane module was polyvinylidene fluoride(PVDF) tubular membrane(Diameter, 12mm;Cutoff molecular weight, 100,000 or so ) obtained from Tianjin Motian Membrane Engineering and Technology Co.Ltd.

In the present experiment, original water after the pretreatment of coagulation sedimentation was added to original 
water tank with the volume of 50L, and then added to hydrolytic acidification bioreactor by diaphragm pump. Wastewater after hydrolytic acidification entered the middle water tank, and after several time's sedimentation came into the biological treatment units of MBR with perforated tube for aeration in the bottom. After that, the mixture went into the tubular membrane module by pump. The module intercepted microorganism and several macromolecular substances which failed to degrade back to the biological treatment units. Permeate liquid discharged as the final effluent of the process.

\section{Results and discussions}

\subsection{Optimization of coagulation sedimentation}

PAC and PFS are the most typical inorganic coagulants, and also coagulant products which were applied extensively and intensively with good effects and cheap price(Malpei, 2003, PP.33-39). In the present study, printing and dyeing wastewater was treated by these two coagulants, and through lab jar test better coagulant was selected.

Effects of these two coagulants were depicted in Figure 2. As seen from Figure 2, under the same addition of 40mg/l, effects of PAC on removal of COD and chroma were significantly better than PFS; As seen from alum blossom settling, the sedimentation speed with the addition of PAC was obviously faster than that of PFS. Therefore, PAC was selected to the coagulant for printing and dyeing wastewater pretreatment.

\subsection{Effects of mixed liquor suspended solids (MLSS) on the function of MBR system}

MLSS is a significant parameter which affected the function of MBR system. Figure 3 depicted the effects of MLSS on removal of chroma under the premise of constant hydraulic retention time $(\mathrm{HRT}=4 \mathrm{~h})$. Removal here included the removal imposed by aerobic biodegradation and interception of membrane itself, as well as the total removal of the whole MBR system.

Removal of chroma by MBR system and biological effects increased initially and then decreased, and didn't vary significantly with the increasing of MLSS. In the early stage of test, with the increasing of MLSS, sludge adsorption effects directly determined the removal effects of chroma, and membrane interception intensified the removal of chroma by MBR system(Brik, 2006, PP.1751-1757), and thus removal of chroma showed a rising trend. With the increasing of MLSS, under the cross-flow operation manner of MBR system, high concentration MLSS was easily shivered which resulted in the reduction of its adsorption function. Furthermore, fine crushing sludge itself would affect the effluents chroma of aerobic units to some extent.

Figure 4 depicted the effects of MLSS on removal of COD under the premise of constant hydraulic retention time $(H R T=4 h)$. Removal of VOD by biological reaction and MBR system showed an increasing trend with the increasing of MLSS, while that by membrane showed an slowly decreasing trend. Thus it could be seen, the removal effects of COD by MBR bioreactor mainly depended on the effects of biological reaction, and membrane only played a certain supplementary and strengthening role in the COD removal, and guaranteed the stability of effluents. Removal of comprehensive chroma and COD attained good effects when aerobic biological treatment units of MLSS was 8g/L, and herein removal of COD by MBR system attained more than $70 \%$.

MBR kept high MLSS and long retention time in the bioreactor by using the separated function of membrane module, and made it have excellent biological treatment effects and resistance capacity to shock loading. However, with the increasing of MLSS, the viscosity, soluble organisms, gel layer, inert substances and so on of mixture would all vary, which could affect membrane flux. Figure 5 depicted that changing rule of membrane flux under different MLSS along with time.

As seen from Figure 5, with the increasing of operation time, membrane flux showed a decreasing trend, but after 10h, the whole decreasing extent of membrane flux didn't not significantly decrease with the increasing of MLSS, and kept within the level of $13 \sim 15 \%$. Strikingly, MLSS played a critical role in initial membrane flux. When MLSS increased from $4.76 \mathrm{~g} / \mathrm{L}$ to $12.11 \mathrm{~g} / \mathrm{L}$, initial membrane flux decreased from $78 \mathrm{~L} / \mathrm{m}^{2} \mathrm{~h}$ to $66 \mathrm{~L} / \mathrm{m}^{2} \mathrm{~h}$, and reduced by $15.4 \%$. Consequently, role of MLSS should be considered not only in the removal of organisms but also the initial membrane flux.

\subsection{Effects of HRT on the function of MBR system}

Figure 6 indicated effects of HRT on the removal of chroma.With the elongation of MBR system HRT, aerobic and the total removal all would increase initially and then decrease, but membrane removal would decrease continuously. Membrane interception only played a supplementary role in the removal of chroma, and with the elongation of biological unit HRT, organic pollutants would be further degraded. However, refractory macromolecular substances would not be degraded with the elongation of HRT, and still remained in the MBR system for further cycles.

Figure 7 depicted effects of HRT on removal of COD. With the elongation of HRT, the removal of COD by biological treatment units increased progressively, and correspondingly, removal of COD by membrane decreased progressively. However, as for the whole system, the removal of COD by MBR system always kept a high value, namely $80 \%$ or so 
when HRT was over $8 \mathrm{~h}$. Consequently, due to the presence of membrane, the quality of effluents was efficaciously guaranteed. In order to keep the stability of MBR system and better effects on removal of comprehensive chroma and COD, HRT of aerobic biological treatment units was selected to be $8 \mathrm{~h}$, which could not only be economic but also meet the requirements of treatment effects.

\subsection{Effects of MBR system on the removal of pollutants at the optimized parameters}

As seen from the previous observations, when aerobic biological treatment units MLSS and HRT was 8g/L and 8h, respectively, effects of MBR system on printing and dyeing wastewater were optimal. Figure 8 indicated effects of MBR system on the removal of organisms at the optimized condition. Obviously, the removal of COD and chroma by tubular MBR system all attained more than $80 \%$. As for the removal of pollutants, MBR biological units played a cardinal role, and membrane interception further strengthened and stabilized effects of MBR system on the removal of chroma and COD.

\subsection{Practical effects of printing and dyeing wastewater treatment}

Compared to the simulated wastewater quality, the quality of practical printing and dyeing wastewater was complicated and fluctuated greatly. Figure 9 indicated the dynamic changing of inlet and outlet chroma and COD.As seen from Figure 9, the changing scope of system inlet and outlet chroma and COD were large, but outlet indices were relatively stable. For chroma, most of them were removed by coagulation treatment, and chroma would not fluctuate greatly when wastewater entered the hydrolytic acidification bioreactor. The following biological treatment units further guaranteed the removal of chroma, which made effluent chroma always lower than 50 in the practical printing and dyeing wastewater treatment. Stable operation of MBR system played a crucial role in the removal of COD, and system effluent COD value was always lower than $100 \mathrm{mg} / \mathrm{L}$, which guaranteed that the effluent quality conformed to the first grade discharge standard of of textile industrial pollutants.

\section{Conclusions}

4.1 Compared to PFS, whether on alum blossom settling or removal of COD, effects of PAC were all better. PAC was used as the optimal coagulants in printing and dyeing wastewater treatment.

4.2 In the certain scope of MLSS, the removal of chroma by MBR system increased with the increasing of MLSS, while decreased when MLSS was excessive high; Removal of COD by MBR system always showed an rising trend with the increasing of MLSS.

4.3 The whole reduction of membrane flux hadn't varied greatly with the increasing of MLSS, while initial membrane flux decreased significantly.

4.4 With the elongation of HRT, removal of COD always showed an increasing trend. But too high HRT was not in favor of the removal of chroma by MBR system.

4.5 The optimal operation conditions in printing and dyeing wastewater treatment by tubular MBR system were as follows: aerobic biological treatment units $M L S S=8 \mathrm{~g} / \mathrm{L}$ and $H R T=8 \mathrm{~h}$. The removal of $C O D$ and chroma by tubular MBR system all attained more than $80 \%$, among which aerobic biological units and membrane accounted for $60 \%$ and $20 \%$ or so, respectively.

4.6 Results of simulated and practical printing and dyeing wastewater treatment indicated that the present process obtained continuous and stable treatment effects, and effluent quality completely conformed to the first grade discharge standard of textile industrial pollutants.

\section{References}

Brik, M., et al. (2006). Advanced treatment of textile wastewater towards reuse using a membrane bioreactor. Process chemistry,41:1751-1757.

Chen, H., \& Gong, M. (2006). Application of hydrolysis-contact oxidation in printing and dyeing wastewater treatment. Technology for Water Treatment, 12:92-94.

Gao, L.Q., Zhang, Y.F., \& Zhang, Z.H. (2008). Membrane technology in the application of textile and dyeing wastewater treatment. Papers of the $8^{\text {th }}$ functional textile and nanotechnology seminar, 4:518-520.

Hao, W.C., Liu, E.H., \& Sun, M., et al. (2005). Study of washing system of a solid-liquid 2-phase tubularmembrane. Technoil of Water treatment, Vo1. 31 No. 2 Feb., PP. 77-80.

Malpei, F., Bonomo, L., \& Rozzi, A. (2003). Feasibility study to upgrade a textile wastewater treatment plant by a hollow fiber membrane bioreactor for effluent reuse.Water Sci.Technol., 47(10)33-39.

Min, Y.A., \& Lu, X.H. (2003). Progress of technology for dyeing wastewater treatment. Industrial Safety and Environmental Protection,29(8):16-19. 
Stephenson, T., Judd, S.J., \& Jefferson, B., et al. (2000). Membrane bioreactors for wastewater treatment. IWA Publishing, London.

Wang, R., Zhang, J., \& Zhang, G. (2006). Hydrolytic Acidification-Biological Contact Oxidation Process for Treatment of Dyeing Wastewater. China Water \& Wastewater, 5:54-55.

Yang, S.M., \& Huang, C.D. (2002). Technology for dyeing industrial wastewater treatment. Beijing: Chemical Industry Press.

Zheng, Y.D., \& Bai, R.C. (1998). Microbial ecology in A/O hydrolysis(acidification) system and its effects on the printing and dyeing wastewater treatment. Environmental Science and Technology, 2;22-23.

Table 1. Water quality simulation of printing and dyeing wastewater

\begin{tabular}{|c|c|c|c|c|}
\hline Chroma & $\operatorname{COD}(\mathrm{mg} / \mathrm{l})$ & $\mathrm{pH}$ & amino-nitrogen $(\mathrm{mg} / \mathrm{l})$ & conductivity $(\mu \mathrm{S} / \mathrm{cm})$ \\
\hline $1800 \sim 2250$ & $797.6 \sim 96.7$ & $10.36 \sim 10.98$ & $23.9 \sim 34.2$ & $765 \sim 1299$ \\
\hline
\end{tabular}



1 original water tank 2 hydrolytic acidification bioreactor 3 aerobic bioreactor 4 Membrane module 5-8 diaphragm pump 9 air pump 10 agitator 11 gas flowmeter 12 liquid flowmeter 13 pressure gauge 14-15 liquid level gauge 16 pipe sparger

Figure 1. Flow chart of experimental apparatus 


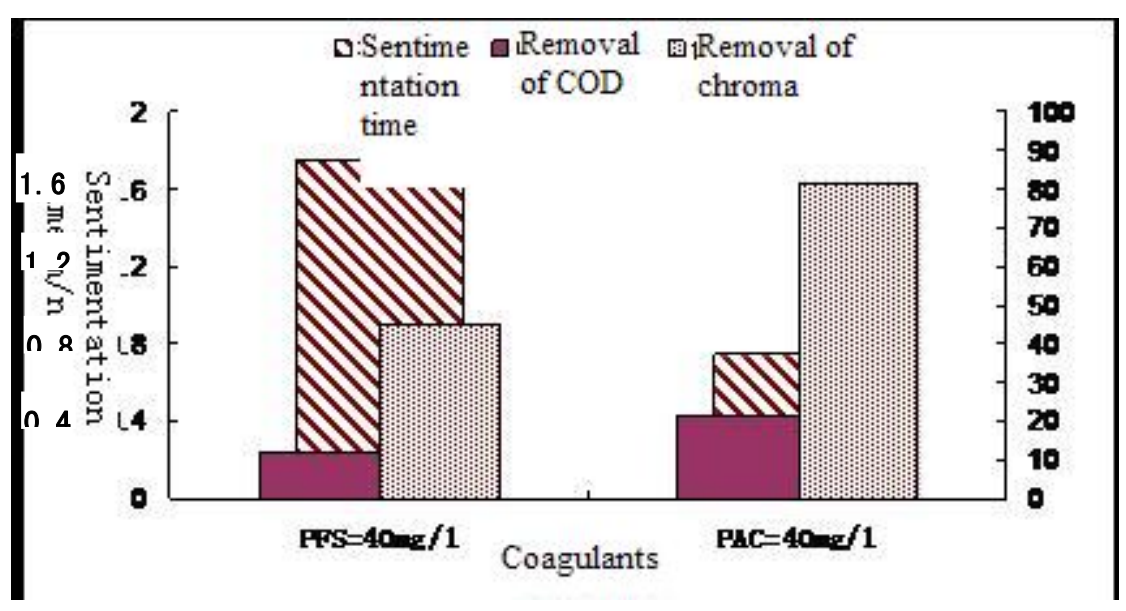

Figure 2. Treatment effects comparison of PAC and PFS

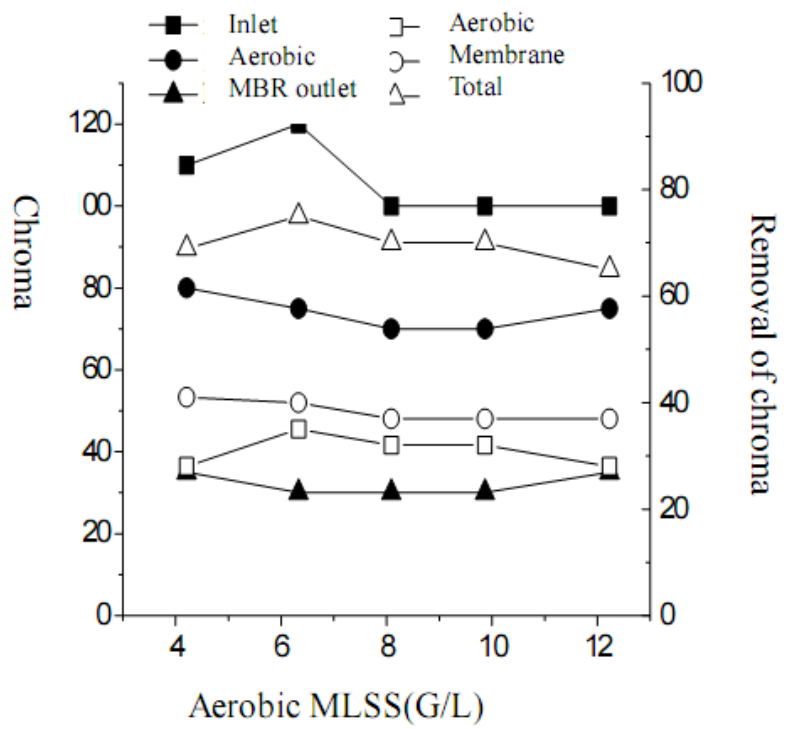

Figure 3. Effects of MLSS on chroma 


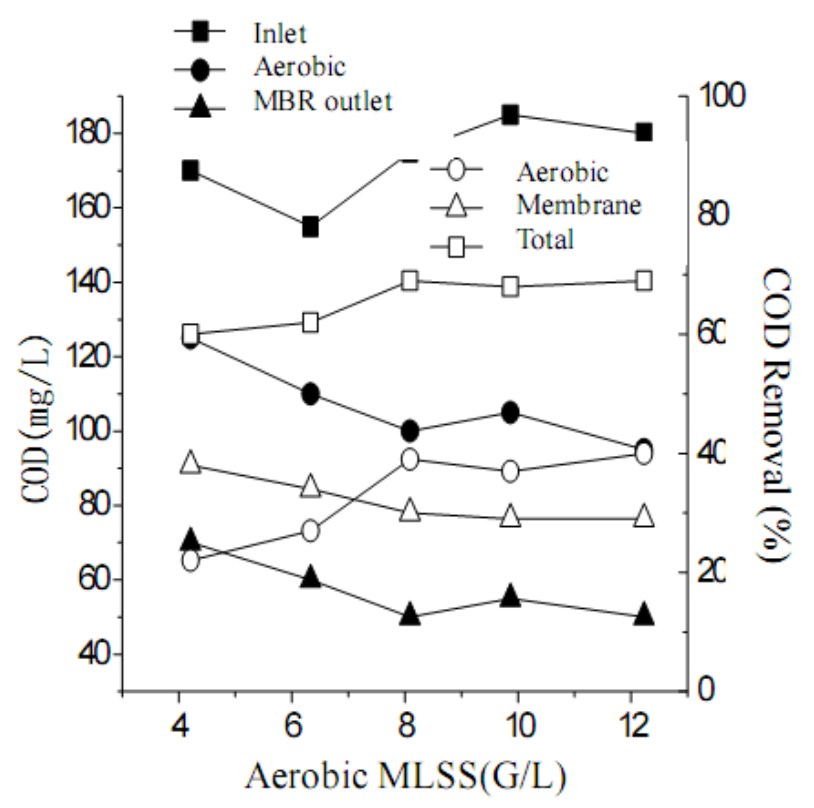

Figure 4. Effects of MLSS on COD

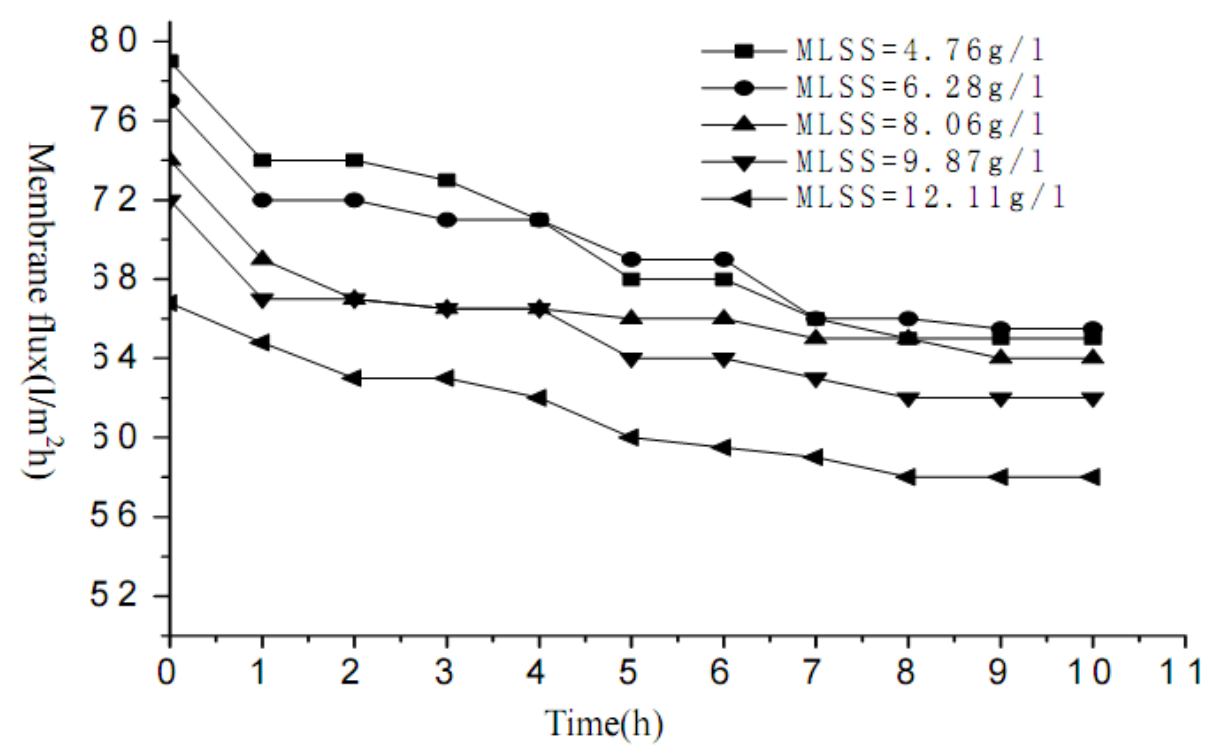

Figure 5. Effects of MLSS on membrane flux 


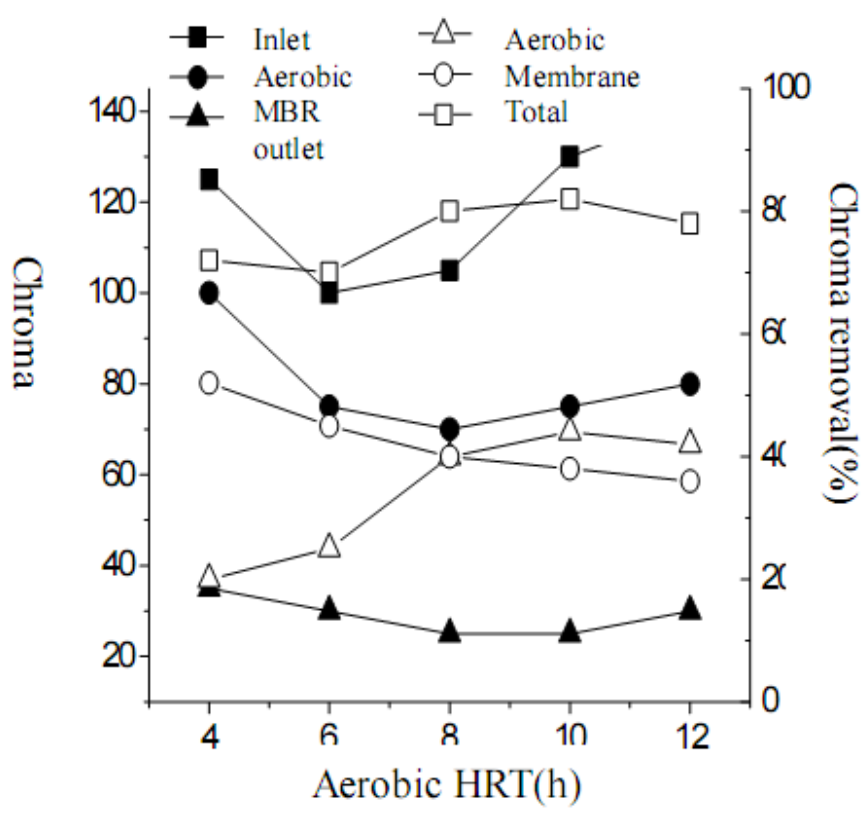

Figure 6. Effects of HRT on chroma

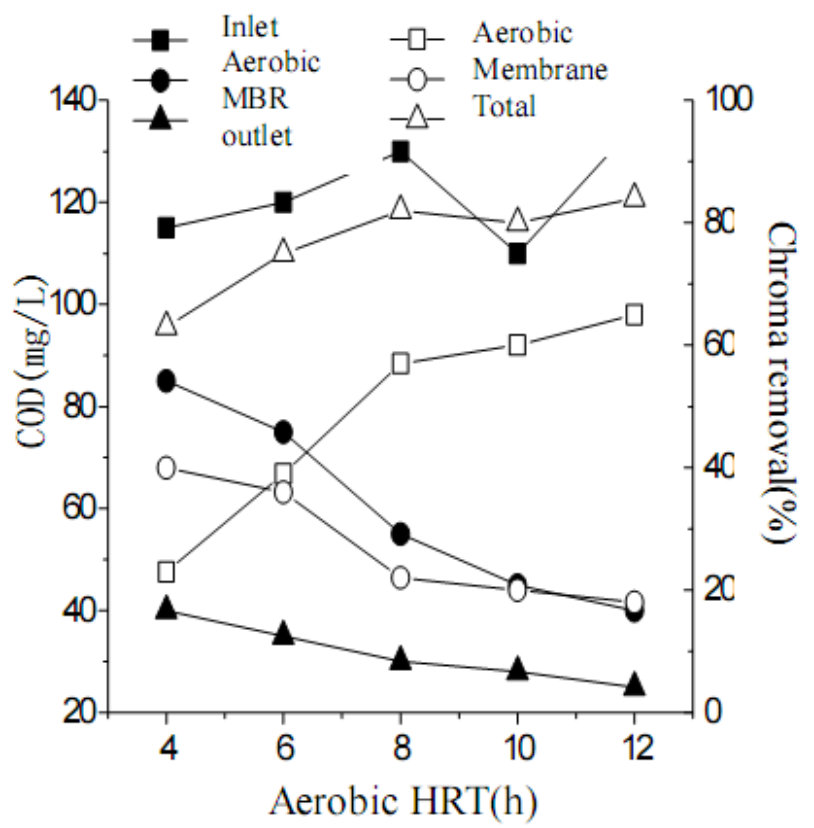

Figure 7. Effects of HRT on COD 


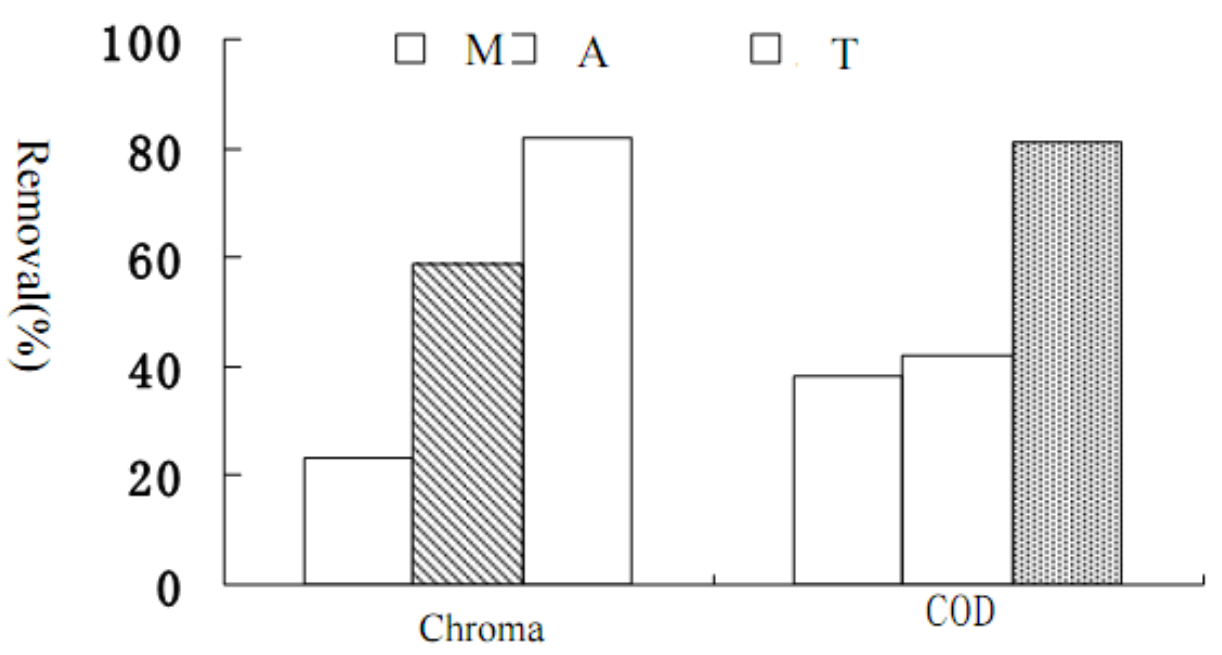

Figure 8. Effects of aerobic sludge and membrane on removal of chroma and COD

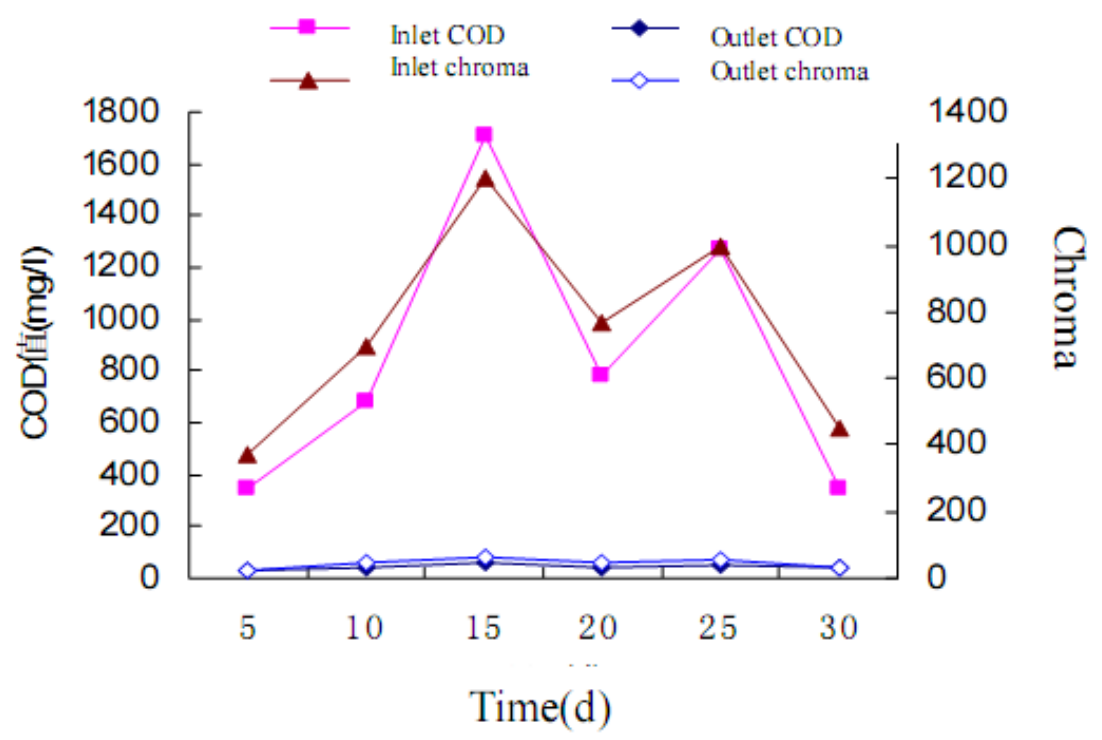

Figure 9. Changing curves of inlet and outlet chroma and COD during the period of experiment 